\title{
A Non-Invasive Method for the Diagnosis of Sjogren's Syndrome with the Evaluation of the Capacity of Salivary Glands
}

\author{
Marina V. Kozlova ${ }^{1}$; Alexander Yu. Vasilyev, $\mathrm{PhD}, \mathrm{ScD}^{2 *}$; Boris A. Arutiunian ${ }^{1}$ \\ ${ }^{I}$ Central State Medical Academy, AD of the President of the RF \\ ${ }^{2}$ Central Research Institute of Radiation Diagnostics \\ Moscow, the Russian Federation
}

\begin{abstract}
This study describes a non-invasive method for diagnosing Sjogren's syndrome (SS) with MR sialography, which is based on the contrasting of the ductal system with its own secretion and the possibility of simultaneous assessment of the secretory function of parotid salivary glands (PSG).

The objective of this study was to evaluate the effectiveness of MR sialography in the diagnosis of SS and chronic parenchymal sialadenitis.

Methods and Results: The study included 60 patients aged between 25 and 70 years with PSG impairments. Inclusion criteria: patients suffering from dry mouth and enlarged PSG, with an established diagnosis of SS and chronic parenchymal sialadenitis. The sensitivity and diagnostic accuracy of MR sialography was $96.6 \%$ and $95.2 \%$ in the diagnosis of SS, and $94.4 \%$ and $93.3 \%$ in the diagnosis of chronic parenchymal sialadenitis. Specificity of the method was $91.6 \%$.

Conclusion: MR sialography, highly effective and identical to sialography, allows detecting typical disorders in parotid salivary glands that are inherent to Sjogren's syndrome and chronic parenchymal sialadenitis. (International Journal of Biomedicine. 2019;9(1):26-30.)
\end{abstract}

Key Words: magnetic resonance imaging $\bullet$ Sjogren's syndrome $\bullet$ parotid salivary glands $\bullet$ parenchymal sialadenitis

\section{Abbreviations}

CPS, chronic parenchymal sialadenitis; PSG, parotid salivary glands; US, ultrasound scan; MRI, magnetic resonance imaging; SS, Sjogren's syndrome.

\section{Introduction}

Among many dental diseases, the prevalence rate of the diseases of the major salivary glands reaches $24 \%$. They include sialolithiasis (36\%), chronic sialadenitis (26\%), and sialadenosis $(18 \%) \cdot{ }^{(1)}$ Sjogren's syndrome (SS) takes a special place in this line with a prevalence of $0.1 \%$ to $3.3 \%$.(2) SS is an autoimmune disease characterized by lymphocytic infiltration of salivary and lacrimal exocrine glands with the development

"Corresponding author: Prof. Alexander Yu. Vasilyev, MD, $P h D, S c D$. Central Research Institute of Radiation Diagnostics. Moscow,Russia.E-mail:auv62@mail.ru of xerostomia and xerophthalmia. SS can be found in 5\%-25\% of patients with systemic diseases of connective tissues, and in $50 \%-75 \%$ of patients with the following autoimmune diseases of liver and thyroid gland: chronic active hepatitis, primary biliary cirrhosis, and Hashimoto thyroiditis. ${ }^{(2,3)}$

According to the criteria classification of SS, introduced by the American-European Consensus Group in 2002, the diagnosis is based on the clinical implications, serological and radiological studies (including sialometry and a Schirmer test), and a biopsy of the minor salivary glands. ${ }^{(4)}$ Additionally, the following examinations can be done: computed tomography, ultrasound scan, and MRI. ${ }^{(5)}$ The most reliable methods recognized to detect typical changes in the glandular tissue 
during SS are salivary gland biopsy and sialograph. ${ }^{\left({ }^{(}\right)}$

However, performing a sialography is often impossible in patients with allergic reactions to the iodine medications and in cases of thyroid diseases, as the administration of the iodine-containing contrast agent is required to perform it. Also, during sialography, PSG are examined only from the one side as performing a dual-sided examination causes additional radiation exposure to the patient. Another important condition that prevents performing the sialography is duct obstruction. ${ }^{(4,7)}$

In these cases, US of the salivary glands is used as a non-invasive method. The main diagnostic criterion of SS during US is parenchymal heterogeneity. This is shown on sonograms in the form of hypoechoic areas surrounded by a hyperechoic line or spot, resembling a reticulated image. ${ }^{(5)}$ At the same time, this complex of symptoms can correspond either to the accumulation of the lymphoid cells or to the finefocal lymphomas and can be mistakenly interpreted. ${ }^{(8)}$

The important data about structural changes of PSG and about the presence and distribution of the adipose tissue can be obtained with an MRI. This is a non-invasive method without radiation exposure, which can also be performed during the recrudescence of sialadenitis. It is usually performed according to the traditional method with a power of $1.0 \mathrm{~T}$ and $1.5 \mathrm{~T}$ without visualization of the ductal system. ${ }^{(3)}$

The objective of this study was to evaluate the effectiveness of MR sialography in the diagnosis of SS and chronic parenchymal sialadenitis (CPS).

\section{Materials and Methods}

The study included 60 patients aged between 25 and 70 years with PSG impairments. Inclusion criteria: patients suffering from dry mouth and enlarged PSG, with an established diagnosis of SS and chronic PS. Exclusion criteria: patients having a pacemaker, metal implants, a salivary gland tumor, or claustrophobia.

Randomization of study groups:

Group 1 included 30 people diagnosed with SS and, depending on the SS stage, was divided into subgroups (Group 1a - 17 patients with a clinically-apparent stage; Group $1 \mathrm{~b}-13$ patients with an advanced stage of a disease).

Group 2 included 18 patients with CPS.

The control group (CG) consisted of 12 volunteers without any salivary gland impairments.

All participants underwent a complex examination to diagnose diseases of PSG.

The secretory function of PSG was evaluated using sialometry. A secretion stimulated by a $2 \%$ ascorbic acid solution was collected for 5 minutes in a graduated tube using a Leshley-Yushchenko-Krasnogorsky capsule.

For sialography, a contrast agent Omnipack-350 was used, which was administered in the ductal system of PSG with the help of the syringe and intravenous plastic catheter, followed by radiography in the anteroposterior and lateral view. MR sialography was performed on a 3.0T GE Signa system.

In the dental office, a duct of PSG was examined with a bougie, followed by obturation with a disposable plastic catheter. After that, stimulation of the salivary discharge with a $2 \%$ ascorbic acid solution was performed for 15 minutes. MRI T1-weighted and T2-weighted scans (cor/sag/ax) and T2- with suppression of the signal from adipose tissue and in FIESTA mode were performed. The slice thickness was $1-5 \mathrm{~mm}$.

The effectiveness of the method was evaluated using the values of diagnostic sensitivity (Se), accuracy ( $\mathrm{Ac}$ ) and specificity $(\mathrm{Sp})$ according to the following formulas:

$$
\text { Se }=\frac{T P}{T P+F N} \times 100 \% \text {, where }
$$

TP - a number of true positive test results in the patients who are known to be ill

FN - a number of false negative results in the patients who are known to be ill

$$
A C=\frac{T P+T N}{T P+T N+F P+F N} \times 100 \% \text {, where }
$$

$\mathrm{TN}$ - a number of true negative results in patients who are known to be healthy

FP - a number of false negative results in patients who are known to be healthy

$$
S p=\frac{T N}{T N+F P} \times 100 \%
$$

Sialography was a referent method.

The quality of the duct visualization on an MR sialogram depends on the amount of salivary secretion in the ducts. To count the volume of the liquid in the ductal system, a formula for calculating the volume of the vessel $\left(\mathrm{Vm}^{3}\right)$ of a cylindrical shape was used. As ducts are visualized on an MR sialogram due to the presence of natural secretion in them, the volume of the vessel (duct) and the volume of liquid in it will be equal.

To find the volume of the vessel, it is necessary to measure its length and radius, while the radius must be constant. Considering that the ductal system of PSG has a different diameter on different sections, first, the volume of the section of the duct with the even radius was determined. For this purpose, the length of the fragment of the duct was measured in the sagittal plane $(\Delta \mathrm{Xi})$ and its radius $\left(\mathrm{r}^{2}\right)$ for this length was measured in the coronal plane.

The volume of the section of the duct $(\Delta \mathrm{Vi})$ was calculated by the following formula:

$\Delta V i=\pi r^{2} \times \Delta X i$, where

$\Delta V i$ - the volume of the section of the duct

$r^{2}$ - the radius of the duct

$\Delta X i$ - the length of the section of the duct

Next, the volume of the duct sections $(\Delta \mathrm{Vi})$ was summed up and the result was the total volume of the vessel $\operatorname{Vi}(\mathrm{m})^{3}$

$V i=\Delta V i_{1}+\Delta V i_{2}+\Delta V i_{3}$

Next, this data was converted into milliliters (Vml), which corresponded to the volume of the liquid in the duct.

In the case of parenchymal parotitis, to obtain reliable data about the amount of the secretion, it is necessary to consider the volume of the liquid, not only in the duct but also in the intraglandular cysts, which are also filled with natural secretion. The following formula for calculatingof the volume of the sphere (cyst) was used: 
$V s=\frac{4}{3} \pi r^{3}$, where

$V_{S}$ - the volume of the cyst

$r^{3-}$ the radius of the cyst

Obtained results of the volume of ducts and volume of cysts were summed, and the result was a total volume of the glandular secretion.

To measure the size of PSG, the special program for calculating the area of figures $\left(\mathrm{Scm}^{2}\right)$ of arbitrary shape IpSquare 5 for Windows was used. The MR-image of PSG in the axial projection was uploaded into the program; the outlines of the parenchyma were traced with the mouse cursor, followed by painting over the gland. The obtained data were transferred to a computer, processed, and the value of the area of the organ appeared at the bottom of the program window.

Statistical analysis was performed using the statistical software «Statistica» (v6.0, StatSoft, USA). Baseline characteristics were summarized as frequencies and percentages for categorical variables and as mean $\pm \mathrm{SD}$ for continuous variables. Inter-group comparisons were performed using Student's t-test. A probability value of $P<0.05$ was considered statistically significant.

\section{Results}

During the examination of CG, the vermilion border had no abnormal elements, PSG were non-palpable. The mucous membrane epithelium of the mouth had a light pink color, moderately moist. With the massage, ducts of PSG released a clear transparent secretion. Sialometry was within a normal range $(4 \pm 0.5 \mathrm{ml})$. There were no abnormal changes detected on sialograms. Parenchyma and ducts were clearly detected, homogeneously filled with a radiopaque contrast agent (Fig.1a). There were no disturbed topographic anatomical criteria on the MR sialogram. PSG were normally positioned and not enlarged $\left(\mathrm{S}=5.5 \pm 0.5 \mathrm{~cm}^{2}\right)$, and had typical shape and structure. Glandular tissue was in a normal condition. Ducts did not have any abnormal changes. Only contours of the ductal system without secretion were visualized without acidic stimulation. After acidic stimulation, it was possible to clearly visualize the ductal system up to second-order ducts. The main duct and first- and second-order ducts with clear even contours were evenly filled with natural secretion along their entire length. The specificity of MR sialography was $91.6 \%$ (Fig.1b).
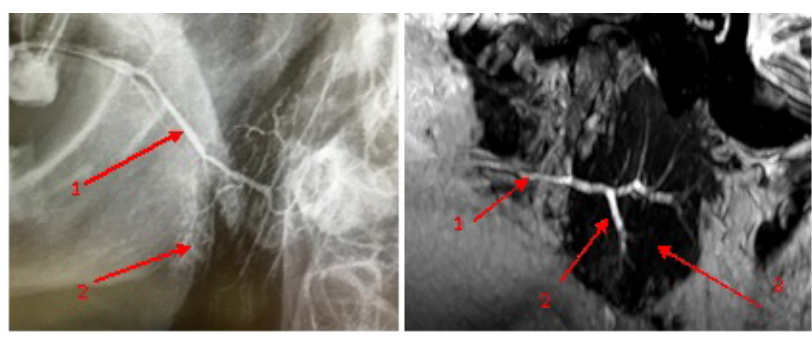

Fig. 1. A 26-year-old patient. Parotid salivary glands. Normal condition.

(1a). Radiographic sialogram (1b). MR sialogram of PSG of PSG on the righ. on the right.

Arrows: 1 -main duct; 2 -ducts of first-and second-order; 3 parenchyma of the gland
Patients of Group 1 complained about dry mouth, the inability to chew food without pre-wetting the mouth with water, about a periodic bilateral enlargement of parotid salivary glands, dry eyes, and joint pain. During a clinical examination, the PSG were found enlarged and painless during palpation; the mucous membrane epithelium of the mouth was dry, hyperemic, free saliva was foaming and was present in low amounts, and multiple non-carious lesions of hard tissue in the area of the necks of the teeth were present.

Patients of Group 1a had xerostomia degree II (sialometry of $1.7 \pm 0.1 \mathrm{ml}$ ). The spotted filling of the parenchyma of the gland with a radiopaque contrast agent was visualized on the sialogram presented in Figure 2a. On an MR sialogram, PSG were enlarged $\left(\mathrm{S}=8.3 \pm 0.3 \mathrm{~cm}^{2}\right)$, parenchyma structure was heterogeneous due to interstitial fibrotic changes, and there was an occurrence of fatty involution and edema. The main duct was clearly and evenly filled with its own secretion along its entire length but only before bifurcation as the secretory function in Group 1a patients was lowered by 2.3 times compared to CC (Fig.2b).
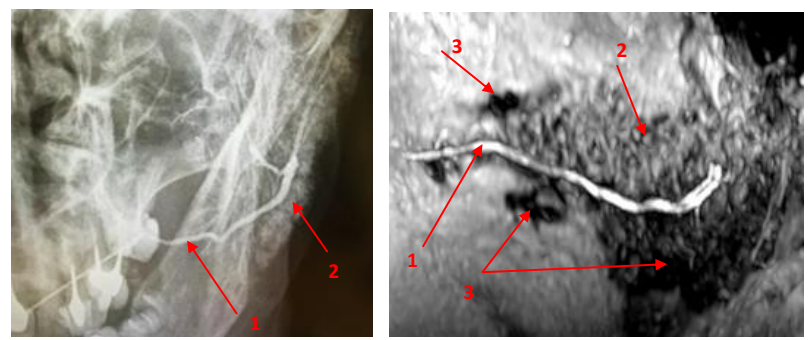

Fig. 2. A 62-year-old patient. SS: clinically-apparent stage.

(2a). Radiographic sialogram of PSG on the left.

(2b). MR-sialogram of PSG on the left. Loss of MR signal.

Arrows: 1 - main duct; 2 - parenchyma of the gland, 3 - interstitial fibrotic changes.

In Group 1b, xerostomia was significantly pronounced, free saliva was absent, and sialometry was $0.7 \pm 0.1 \mathrm{ml}$ (xerostomia degree III). Cyst-like cavities in the form of "clouds" with fuzzy blurred outlines were found on the sialogram presented in Figure 2, which indicates an increased permeability of the gland tissue. The third- and fourth-order ducts cannot be tracked, local sections of strictures and ectasias of first- and second-order ducts were present (Fig.3a).
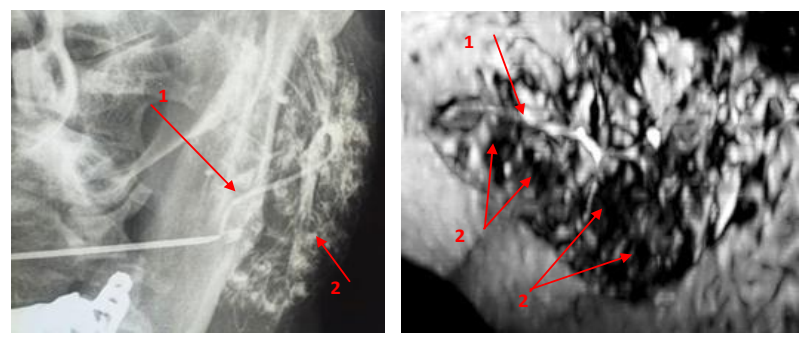

Fig. 3. A 65-year-old patient. The advanced stage of SS. (3a). Radiographic sialogram of PSG on the left.

(3b). MR-sialogram of PSG on the left. Loss of MR signal.

Arrows: 1-main duct; 2 - parenchyma of the gland, 3- fibrocystic adipose changes.

On the MR sialograms of the advanced stage of SS, PSG were found to be enlarged $\left(\mathrm{S}=6.6 \pm 0.1 \mathrm{~cm}^{2}\right)$, and parenchyma 
had diffuse cellular restructuring due to the pronounced fibrocystic adipose changes (the consequences of destructive changes). The ductal system can be traced only partially, which is indicated by a sharp decrease in the PSG secretion. The MR image corresponds to structural changes in the salivary glands (Fig. 3b). The sensitivity and diagnostic accuracy of MR sialography in the diagnosis of SS were $96.6 \%$ and $95.2 \%$, respectively.

In Group 2, PSG were enlarged on both sides, soft and elastic in consistency under palpation, and painless. During the examination of the oral cavity, a mucous membrane was found to be poorly moistened, wedge-shaped defects of teeth were found, and ducts released cloudy secretion. Sialometry was $2.2 \pm 0.2 \mathrm{ml}$, which corresponds to xerostomia stage I. The sialogram presented in Figure 4a shows signs of CPS in the form of cystic cavities filled with a radiopaque contrast agent more than $1 \mathrm{~mm}$ in diameter. The MR sialogram presented in Figure $4 \mathrm{~b}$ shows bilateral enlargement of PSG $\left(\mathrm{S}=6.7 \pm 0.1 \mathrm{~cm}^{2}\right)$ with numerous small intraglandular cysts of different sizes, filled with natural secretion, and also fibrosis changes of the interstitial tissue of the gland. The sensitivity and diagnostic accuracy of MR sialography in the diagnosis of CPS were $94.4 \%$ and $93.3 \%$, respectively.
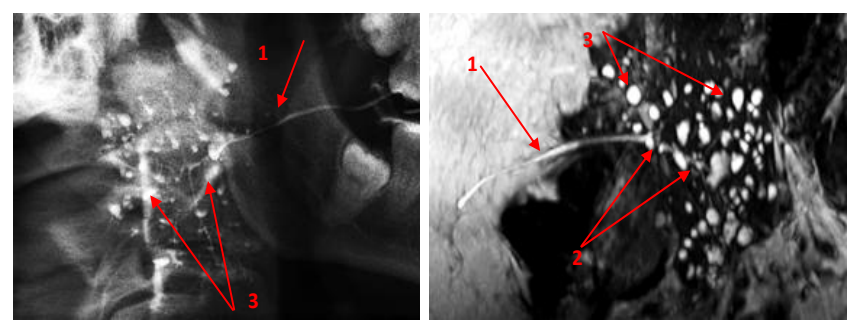

Fig. 4. A 38-year-old patient. Chronic bilateral parenchymal sialadenitis.

(4a). Radiographic sialogram of PSG on the right.

(4b). MR sialogram of PSG on the right.

Arrows: 1 - main duct; 2 -ducts of the II order; 3 -cysticshaped cavities.

\section{Results and Discussion}

During analysis of MR sialograms and data received from sialometries, it was possible to determine a verifiable relation between the secretion level of PSG and the visualization degree of the ductal system of glands during MR sialography after acidic stimulation for salivary discharge. As salivation decreased, the volume of secretion in the ducts also decreased, which led to a worse tracing of the architectonics of the salivary gland ducts (Table 1).

Using the program for calculating the area of figures of arbitrary shape, IpSquare 5 for Windows, allowed us to determine the true size of parotid salivary glands in their normal condition and when affected by different diseases. It was established that PSG in the clinically-apparent stage of SS are enlarged by 1.5 times. With the advanced stage and PS, PSG are enlarged 1.2 times compared to the normal condition $(P<0.05)$. This may be caused by the pronounced destructive processes in the form of fibrous involution of glandular tissue (Fig.5).
Table 1.

Data of MR sialograms and sialometry in the examined groups

\begin{tabular}{|l|c|c|}
\hline \multicolumn{1}{|c|}{ Group } & $\begin{array}{c}\text { Sialometry } \\
\text { (amount of saliva, ml) }\end{array}$ & $\begin{array}{c}\text { MR sialogram } \\
\text { (volume of secretion } \\
\text { in the duct, V ml) }\end{array}$ \\
\hline CG & $4.0 \pm 0.5$ & $2.1 \pm 0.3$ \\
\hline Group 2 & $\begin{array}{c}2.2 \pm 0.2^{*} \\
\text { xerostomia degree I }\end{array}$ & $1.2 \pm 0.2^{*}$ \\
\hline Group 1a & $\begin{array}{c}1.7 \pm 0,1^{*} \\
\text { xerostomia degree II }\end{array}$ & $0.5 \pm 0.1^{*}$ \\
\hline Group 1b & $\begin{array}{c}0.7 \pm 0.1^{*} \\
\text { xerostomia degree III }\end{array}$ & $0.2 \pm 0.1^{*}$ \\
\hline
\end{tabular}

$*_{-} P<0.05$

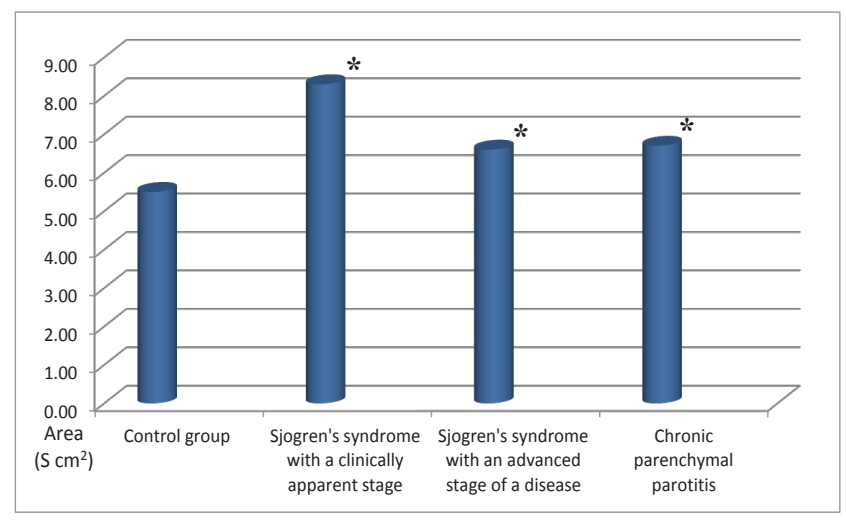

Fig. 5. Diagram of the area $\left(\mathrm{S} \mathrm{cm}^{2}\right)$ of $P S G$ affected by different diseases $\left(*_{-} P<0.05\right)$.

\section{Conclusions:}

-MR sialography, highly effective and identical to sialography, allows detecting typical disorders in PSG that are inherent to SS and CPS.

-The sensitivity and diagnostic accuracy of MR sialography was $96.6 \%$ and $95.2 \%$ in the diagnosis of SS, and $94.4 \%$ and $93.3 \%$ in the diagnosis of CPS. Specificity of the method was $91.6 \%$.

-Direct relations between the secretion level of PSG and the visualization of the ductal system on MR sialograms were established, which allows using MR sialography as a functional method of evaluating the secretory function of PSG

-To determine the sizes of PSG, it is recommended to measure their area in axial projection using the program IpSquare 5 for Windows.

\section{Conflict of Interest} interests.

The authors declare that they have no competing

\section{References}

1. Afanasev VV, Khubutia BN, Vinokurova OY, Denisova EI. [Structure of morbidity of the salivary glands according 
to the data of the All-Russian center for the study of diseases of the salivary glands on the basis of the clinic dental surgery maxillo-facial hospital of war veterans of the department of healthcare of Moscow]. Russian Journal of Dentistry. 2012;(6):36-37. [Article in Russian].

2. Vasilyev AYu. [Clinic, diagnosis and differential diagnosis of Sjogren's disease]. Russ Med Zhurnal. 2008;(10):638-648. [Article in Russian].

3. Ren YD, Li XR, Zhang J, Long LL, Li WX, Han YQ. Conventional MRI techniques combined with MR sialography on T2-3D-DRIVE in Sjögren syndrome. Int J Clin Exp Med. 2015;8(3):3974-82.

4. Vitali C, Bombardieri S, Jonsson R, Moutsopoulos HM, Alexander EL, Carsons SE, et al; European Study Group on Classification Criteria for Sjögren's Syndrome. Classification criteria for Sjögren's syndrome: a revised version of the European criteria proposed by the American-European Consensus Group. Ann Rheum Dis. 2002;61(6):554-8.
5. Saied F, Włodkowska-Korytkowska M, Maślińska M, Kwiatkowska B, Kunisz W, Smorawińska P, Sudoł-Szopińska I. The usefulness of ultrasound in the diagnostics of Sjögren's syndrome. J Ultrason. 2013;13(53):202-211. doi: 10.15557/ JoU.2013.0020.

6. Niemelä RK, Pääkkö E, Suramo I, Takalo R, Hakala M. Magnetic resonance imaging and magnetic resonance sialography of parotid glands in primary Sjögren's syndrome. Arthritis Rheum. 2001;45(6):512-8.

7. Karaca Erdoğan N, Altay C, Özenler N, Bozkurt T, Uluç E, Dirim Mete B, Özdemir I. Magnetic resonance sialography findings of submandibular ducts imaging. Biomed Res Int. 2013;2013:417052. doi: 10.1155/2013/417052.

8. Kozlova MV, Vasilyev AYu, Arutiunian BA, Repin IS. [Improvement of radiological methods for diagnosing diseases of the large salivary glands]. Kremlevskaja Medicina. Klinicheskij Vestnik. 2017;(3):19-23. [Article in Russian]. 